\title{
A Single Blind Comparative Randomized Non-Inferior Multicenter Study for Efficacy and Safety of Levofloxacin versus Ciprofloxacin in the Treatment of Uncomplicated Typhoid Fever*
}

\author{
Ronald Nelwan', Khie Chen Lie ${ }^{1}$, Suharyo Hadisaputro ${ }^{2}$, Eddy Suwandoyo ${ }^{3}$, Suharto ${ }^{3}$, \\ Nasronudin $^{3}$, Hadi Yusuf ${ }^{4}$, Primal Sudjana ${ }^{4}$, Gatoet Ismanoe ${ }^{5}$, Djoni Djunaedi ${ }^{5}$, \\ Halim Mubin ${ }^{6}$, Munirah Said ${ }^{6}$, Diana Paramita ${ }^{7}$ \\ ${ }^{1}$ Divisions of Tropical \& Infectious Diseases, University of Indonesia, Jakarta, Indonesia \\ ${ }^{2}$ Divisions of Tropical \& Infectious Diseases, Diponegoro University, Semarang, Indonesia \\ ${ }^{3}$ Divisions of Tropical \& Infectious Diseases, Airlangga University, Surabaya, Indonesia \\ ${ }^{4}$ Divisions of Tropical \& Infectious Diseases, Padjajaran University, Bandung, Indonesia \\ ${ }^{5}$ Divisions of Tropical \& Infectious Diseases, Brawijaya University, Malang, Indonesia \\ ${ }^{6}$ Divisions of Tropical \& Infectious Diseases, Hasanudin University, Makassar, Indonesia \\ ${ }^{7}$ Department of Internal Medicine, Persahabatan Hospital, Jakarta, Indonesia \\ Email: jade_update@yahoo.com
}

Received November 2, 2012; revised December 3, 2012; accepted January 5, 2013

\begin{abstract}
Ciprofloxacin is currently the drug of choice for typhoid fever, but Salmonella typhi resistance to ciprofloxacin is increasing, while levofloxacin has been shown to be very effective in a few open studies. This study aimed to compare the efficacy, and safety of levofloxacin and ciprofloxacin for typhoid fever. From 110 patients with confirmed typhoid fever, 54 patients received oral levofloxacin $500 \mathrm{mg}$ once daily with one drop out, while 56 received ciprofloxacin 500 $\mathrm{mg}$ twice daily for 7 days with two drop outs. Defervescence of fever was achieved on an average of 3 days after initiating levofloxacin and 5 days after starting ciprofloxacin and one microbiologically non confirmed typhoid fever relapse occurred in the levofloxacin group while two relapses with positive Salmonella microorganism occurred in the ciprofloxacin group. No carrier of Salmonella typhi was found in both groups at day 30. Adverse reactions were more pronounced in the ciprofloxacin group compared to the levofloxacin group. In conclusion, oral levofloxacin $500 \mathrm{mg}$ once daily for one week showed faster fever clearance compared to ciprofloxacin $500 \mathrm{mg}$ twice daily in typhoid fever in Indonesia, and less adverse reactions occurred with levofloxacin compared to ciprofloxacin. This electronic document is a "live" template. The various components of your paper (title, text, heads, etc.) are already defined on the style sheet, as illustrated by the portions given in this document.
\end{abstract}

Keywords: Typhoid Fever; Levofloxacin; Ciprofloxacin; Comparative Clinical Study; Indonesia

\section{Introduction}

Typhoid fever is still a big threat to the communities in many developing countries; it contributes to acute morbidity and also mortality [1]. The multidrug resistant strains of Salmonella typhi characterized by resistance to chloramphenicol, ampicillin, co-trimoxazole were reportedly to be increased since the early nineties [2]. Excellent results were recorded and even faster relief of signs and symptoms of typhoid fever was obtained by using fluoroquinolones, and most significantly post-treatment

*Administration support: Dr. Felisia Ariyani Santoso and Dr. Suyanto, Daiichi Sankyo Co. Ltd. Jakarta Representative Office. carrier rates were near to zero so it was soon adopted as the best option to be used in patients with this kind of infection. The drug of choice used around the world at this moment is ciprofloxacin that has emerged as an excellent alternative for treatment of typhoid fever [3]. However, as is nowadays acknowledged, there is a rise in ciprofloxacin resistant Salmonella typhi strains with perhaps more unfavorable outcomes in duration of morbidity and also in causing mortality [4-6]. In Indonesia, as early as 1996, a case of clinical failure due to resistance of Salmonella typhi to ciprofloxacin was experienced and reported [7]. Even an appeal has been made to reconsider ciprofloxacin to be used for typhoid fever [8]. On the 
other hand, levofloxacin, the I-isomer of the racemic ofloxacin, that itself is also a potent inhibitor of Salmonella typhi infections [9], was shown to be very effective with excellent results as reported in an open study [10]. The aim of the present study was to compare the clinical and bacteriological efficacy, tolerability and safety between levofloxacin and ciprofloxacin in a single blind randomized study for the treatment of uncomplicated typhoid fever.

\section{Method and Patients}

\subsection{Study Design}

This single blind comparative randomized multi center trial was conducted in 2006 and 2007 in the Divisions of Tropical and Infectious Diseases Faculty of Medicine respectively of the Universities of Indonesia (Jakarta), Padjajaran (Bandung), Diponegoro (Semarang), Airlangga (Surabaya), Brawijaya (Malang), and Hasanudin (Makassar). Patients were recruited from the main University's Hospital, Dr. Cipto Mangunkusumo (Jakarta), Hasan Sadikin Hospital (Bandung), dr Kariadi Hospital (Semarang), Dr. Soetomo Hospital (Surabaya), Dr. Saiful Anwar Hospital (Malang), Dr. Wahidin Sudirohusodo Hospital (Makassar), as well as those with close affiliation like the Persahabatan Hospital, Jakarta. The study was reviewed and approved by the Ethics Committee of the University of Indonesia.

\subsection{Study Population}

Patients who were admitted to either one of the participating medical centers with the suspicion of suffering from uncomplicated typhoid fever were screened for inclusion in this study. In order to indentify candidate to be included properly, the patient was subjected to a clinical score developed by the Division of Tropical and Infectious Diseases Faculty of Medicine University of Indonesia that has been used for about a decade and a half, helping to determine whether a patient should go through the baseline clinical and laboratory evaluation or not. This clinical score will select a patient on a percentage basis for possibility having typhoid fever. A scoring point of one is given to the following symptoms: fever less than one week, headache, weakness, nausea, abdominal pain, anorexia, vomiting, disturbed GI motility, insomnia, hepatomegaly, and splenomegaly. A two point score will be applied to: fever more than one week, relative bradycardia, typhoid tongue, melena stools, and impaired consciousness. The lower the score the less chance for suffering from typhoid fever infection. A clinical score of 13 or more is most likely to be positive for Salmonella typhi infections, while a clinical score of around 10 will give a fifty percent chance to be positive. Patients with a clinical score of 7 or less have a very limited chance of suffering from typhoid fever as seen in Table 1 [11].
The inclusion criteria were male or female with an age ranged of $18-65$ years with the precaution that females in childbearing age should have negative pregnancy test and should use adequate contraception during the study period. Non-childbearing potential female patients included post menopausal, surgically sterilized or having a hysterectomy 3 months prior to the study. Patients included had at least 3 days duration of fever and no longer than 20 days. A total clinical score of 8 or more should be obtained by the patients for inclusion.

The exclusion criteria were increased creatinine values, a history of adverse reactions to quinolones, a life threatening infection or serious underlying disease, a history of convulsions or photosensitive reactions to medication or being on theophylline or warfar in medication. A clinical score of 16 or above rendering the possibility of complicated typhoid fever were also excluded. Patients with prior use of anti typhoidal preparations were also excluded. Also patients who could not swallow the medication due to severe vomiting or were suffering from serious diarrhea were excluded.

After the nature of the study was explained, the patient was requested to sign informed consent forms when they agreed to take part in the study.

Table 1. Clinical score for typhoid fever.

\begin{tabular}{ccc}
\hline No. & Sign and/or symptom & Score \\
\hline 1 & Fever $<1$ week & 1 \\
2 & Headache & 1 \\
3 & Weakness & 1 \\
4 & Nausea & 1 \\
5 & Abdominal pain & 1 \\
6 & Anorexia & 1 \\
7 & Vomiting & 1 \\
8 & Disturbed GI motility & 1 \\
9 & Insomnia & 1 \\
10 & Hepatomegaly & 1 \\
11 & Splenomegali & 1 \\
12 & Fever $>1$ week & 2 \\
13 & Relative bradycardia & 2 \\
14 & Typhoid tongue & 2 \\
15 & Melena stools & 2 \\
16 & Impaired consciousness & 2 \\
\hline
\end{tabular}

${ }^{*}$ The total score has a maximum of 20 . If the total score is $\geq 13$, the patient is most likely to suffer from typhoid fever. If the total score is $8-12$, the patient has $50 \%$ chance of suffering from typhoid fever. If the total score is $\leq 7$, the patient has very limited chance of suffering from typhoid fever. 


\section{Procedures}

Baseline clinical manifestation were obtained through the scoring table while baseline laboratory examination included routine haematology, blood chemistry, Widal agglutination test, urinalysis, C-reactive protein, stool and blood culture and PCR (Polymerase Chain Reaction) to detect Salmonella typhi as it was shown to be effective in diagnosis of problematic cases [12]. Method of laboratory tests for haemoglobin, haematocrit, leucocyte count, and leucocyte differential count, and also sedimentation was done with SYSMEX (according to International Council for Standard Haematology methods). Total bilirubin was determined by Dichloraniline method, while creatinine used JAFFE compensated rate. For AST and ALT, determined according to International Federation of Chemical Chemistry method. All combined in the VITROS system. Immunoturbidimetry was used for Widal serology test and urine albumin urinalysis. While refractometry was used for erythrocyte, leucocyte, cast/ bacteria, crystal/epitel counts in urine analysis. For blood and stool culture, we used standard media culture for Salmonella.

Patients were randomized to receive either oral levofloxacin $500 \mathrm{mg}$ with identical placebo administered twice daily or $500 \mathrm{mg}$ oral ciprofloxacin also administered twice daily for the total duration of 7 days. All were given in sealed envelopes, so that the investigator did not know which medication would be given to which patient. A daily medical examination was performed to obtain clinical status and to detect any possible adverse reactions. On day 8 all laboratory examination screened at day 1 except for PCR were repeated. Patients who did not respond to treatment by day 8 were continued by the presently recommended treatment of ciprofloxacin 500 $\mathrm{mg}$ bid. For concomitant treatment no other antimicrobial drug was allowed. For other medication during the course of study, date of administration and dosage/duration of its usage was recorded on the case report form. Antipyretics were the only drugs given for hyperpyrexia.

On day 38 - 45, approximately one month after the last day of treatment, the patients were asked to return for a physical examination and a stool examination for detection of S typhi carrier state. Any clinical relapse of fever after hospital discharge was instructed to be reported as soon as possible.

\subsection{Diagnosis of Typhoid Fever}

Clinical presentations suggestive for a Salmonella typhi infection and supported by a clinical score were either confirmed by microbiology culture showing positive $S$. typhi in the blood, or by Polymerase Chain Reaction or serology by Widal agglutination showing a fourfold increase of titer in two successive measurement one week apart or with two successive high titer, $\geq 1 / 640$ in measurements of $S$. typhi $\mathrm{O}$ and $\geq 1 / 1280$ for $S$. typhi $\mathrm{H}$ [13, $14]$.

\subsection{Sample Size Calculations}

Estimation for number of recruited patients in this study was calculated based on equation for equivalence trial:

$$
N=\frac{2 P Q(Z 1-\alpha)^{2}+(Z 1-\beta)^{2}}{d^{2}}
$$

it was assumed that the efficacy of levofloxacin and ciprofloxacin is the same for treatment of typhoid fever $(P)$ $=98 \%$.

Based on:

$Q=1-P$

$N=$ estimate number of patients/arm

$\alpha=5 \%$ (2 tailed) $\rightarrow Z 1-\alpha 2=Z 97.5 \%=1.96$

$\beta=10 \%$ (1 tailed) $\rightarrow Z 1-\beta=Z 90 \%=1.282$ (power $10 \%)$

$d=$ maximum difference which has no clinical significance (clinical judgement) $=10 \%$

$N=\left(2 \times 0.98 \times 0.02 \times(1.96+1.282)^{2}\right) /(0.10 \times 0.10)=$ 42 patients definite case of thypoid/arm

Because from the scoring experience of $\geq 8$ points where the rate of definite cases was $40 \%$, the total number of cases typhoid fever that would be needed for each arm would be: $100 / 40 \times 42=106$ patients.

\subsection{Outcome}

The primary end point was the 7 th day cure rate. The patient was expected to be free from fever and no positive Salmonella typhi present in the blood or stool on culture. The occurrence of a possible carrier state was determined one month after the patient finished the treatment with assessment of stool culture for $S$. typhi. Clinical relapse was recorded when patient had recurrence of fever during this one month period after treatment.

\subsection{Data Analysis}

Descriptive statistics was calculated for continuous variables. Geometric means was calculated for baseline characteristics and applied to clinical resolution of fever and recorded adverse reactions.

\section{Results}

\subsection{Patient Characteristics}

Two hundred and twelve cases were enrolled during the study period. All cases were suspected of suffering from acute uncomplicated typhoid fever. Among the 212 cases typhoid fever was confirmed in a total of 110 cases. Ten patients dropped out for various legitimate reasons. From 
the 202 remaining patients, 110 fulfilled criteria of final typhoid fever diagnosis based on either positive microbiologic culture for S. typhi, positive PCR for S. typhi or significant four fold rise in agglutination according to the Widal agglutination test or with high $(\geq 1 / 640)$ titers for $S$. typhi $\mathrm{O}$ and $\geq 1 / 1280$ titers for $S$. typh $\mathrm{H}$. In the other 92 patients, typhoid fever was not confirmed by laboratory test and therefore excluded from the final analysis. However all 212 patients were included for clinical safety and tolerability comparing levofloxacin to ciprofloxacin.

From the 110 patient in the final analysis 54 belonged to the levofloxacin group while 56 were in the ciprofloxacin arm. Three patients dropped out in this confirmed $S$. typhi infection group. Reasons for dropped out were intolerability to oral intake in the ciprofloxacin arm while there was an underlying positive HIV infection in the levofloxacin arm.

The demographic comparison between the two groups did not show any statistical difference with regard to gender or age (see Table 2). On the same table can also be noted that there was also no difference with regard to the mean duration of fever in both groups as well as the obtained initial mean clinical score for typhoid fever. The method of diagnosis in both arm are outlined in Table 3. After administration of the medication as outlined in the study protocol defer vescence of fever was achieved on an average of 3 days in the levofloxacin group and on the average of 5 days in the ciprofloxacin group.

Table 2. Baseline characteristics of patients in levofloxacin and ciprofloxacin groups.

\begin{tabular}{ccc}
\hline \multirow{2}{*}{ Variable } & \multicolumn{2}{c}{ No. of patients } \\
\cline { 2 - 3 } & Levofloxacin & Ciprofloxacin \\
\cline { 2 - 3 } & $(\mathrm{n}=53)$ & $(\mathrm{n}=54)$ \\
\hline Male-female ratio & $26: 27$ & $25: 29$ \\
Mean age (range) & $25.1(18-53)$ & $26.6(18-54)$ \\
Mean clinical score & 10.7 & 9.8 \\
Mean duration of fever (days) & 7.7 & 7.5 \\
\hline
\end{tabular}

Table 3. Typhoid fever diagnosis in levofloxacin and ciprofloxacin groups.

\begin{tabular}{ccc}
\hline \multirow{2}{*}{ Diagnosis } & \multicolumn{2}{c}{ No. of patients } \\
\cline { 2 - 3 } & Levofloxacin & Ciprofloxacin \\
\cline { 2 - 3 }$(\mathrm{n}=54)$ & $(\mathrm{n}=56)$ \\
\hline Microbiology culture & 11 & 14 \\
Polymerase chain reaction & 19 & 24 \\
Serology (widal test) & 23 & 2 \\
Dropout(s) & 1 & \multicolumn{2}{c}{ (s) }
\end{tabular}

* One week consecutive $S$. typhi titer $\mathrm{O} \geq 640, \mathrm{H} \geq 1280$ or 4 -fold increase.
An overall assessment of clinical efficacy can be noted in Table 4 where absence of fever in the levofloxacin group was achieved by day 7 while this condition was achieved only in $77.8 \%$ of the patients in the ciprofloxacin group. There were three relapses in this study, one belonging to the levofloxacin group and two to the ciprofloxacin group where was identified a case of Salmonella arizonae which was the same as the initial infection. It was also interesting to note that in the cases of clinical relapse in the ciprofloxacin group the microbiology could be confirmed which was not the case in the levofloxacin arm. Parallel to the late resolution in the ciprofloxacin group there were still positive $S$. typhi on culture from blood and stool after the 7 th end of treatment day (Table 5).

Table 6 is describing about adverse reactions in the two groups of patients was more pronounced in the ciprofloxacin group where nausea and vomiting together dominated and where a few patients also complaint of epigastric pain/distress while for central nervous system adverse reactions, patients suffering from insomnia/cephalgia the rate was almostequal in both group ( 7 in levofloxacin arm and 6 in ciprofloxacin arm).

Unwanted laboratory reactions with more than a 3 times increase of liver transsaminase (SGPT) was more pronounced in the ciprofloxacin group occurring in six cases while there were only two with this kind of adverse laboratory reaction in the levofloxacin group for the

Table 4. Clinical efficacy of levofloxacin vs ciprofloxacin for typhoid fever.

\begin{tabular}{lccc}
\hline Clinical efficacy & Levofloxacin & Ciprofloxacin & $\begin{array}{c}\text { Levofloxacin vs } \\
\text { ciprofloxacin }\end{array}$ \\
\cline { 2 - 4 }$(\mathrm{n}=53)$ & $(\mathrm{n}=54)$ & $5.0(3.60)$ & $\begin{array}{c}\mathrm{K}=1.81 \\
(\mathrm{p}<0.005)\end{array}$ \\
$\begin{array}{c}\text { Mean (SD) days of } \\
\text { defervescence }\end{array}$ & $3.0(0.96)$ & $42 / 54(77.8 \%)$ & $\begin{array}{l}\mathrm{X}^{2}=11.13 \\
(\mathrm{p}<0.001)\end{array}$ \\
Fever free on day 7 $53 / 53(100 \%)$ & $2^{*}$ & \\
Clinical relapse & $1^{* *}$ &
\end{tabular}

"Salmonella arizonae in 1 patient and S. typhi in the other patient; ${ }^{* *}$ Salmonella not identified.

Table 5. Post treatment microbiological culture levofloxacin vs ciprofloxacin for typhoid fever.

\begin{tabular}{ccc}
\hline & \multicolumn{2}{c}{ No. of patients } \\
\cline { 2 - 3 } Microbiological culture & Levofloxacin & Ciprofloxacin \\
\cline { 2 - 3 }$(\mathrm{n}=53)$ & $(\mathrm{n}=54)$ \\
\hline $\begin{array}{c}\text { Culture of } S . \text { typhi }(+) \text { on } \\
\text { day } 7 \text { (blood) }\end{array}$ & none & $8(14.8 \%)$ \\
$\begin{array}{c}\text { Culture of } S . \text { typhi }(+) \text { on } \\
\text { day } 7 \text { (stool) } \\
\begin{array}{c}\text { Bacteriological relapse } \\
\text { post treatment }\end{array}\end{array}$ & none & $4(7.4 \%)$ \\
Carrier at day 30 & none & 1 case \\
\hline
\end{tabular}


Table 6. Comparison of adverse reactions in levofloxacin and ciprofloxacin groups in confirmed typhoid fever patients and in all patients.

\begin{tabular}{|c|c|c|c|c|}
\hline \multirow{2}{*}{ Adverse reactions } & \multicolumn{2}{|c|}{$\begin{array}{l}\text { No. of patients } \\
\text { (typhoid fever) }\end{array}$} & \multicolumn{2}{|c|}{$\begin{array}{l}\text { No. of patients } \\
\text { (All) }\end{array}$} \\
\hline & Levofloxacin $(\mathrm{n}=53)$ & Ciprofloxacin $(\mathrm{n}=54)$ & Levofloxacin $(\mathrm{n}=103)$ & Ciprofloxacin $(n=109)$ \\
\hline Nausea $(\mathrm{N})$ & 5 & 4 & 8 & $9^{*}$ \\
\hline Vomiting (V) & 1 & 2 & 1 & 2 \\
\hline $\mathrm{N} \& \mathrm{~V}$ & 0 & 4 & 1 & $6^{*}$ \\
\hline Epigastric distress/pain & 0 & 2 & 0 & 3 \\
\hline N-V-Insomnia & 0 & 0 & 1 & $1^{*}$ \\
\hline N-Insomnia & 0 & 0 & 3 & $2^{*}$ \\
\hline Insomnia & 4 & 2 & 7 & 5 \\
\hline Cephalgia & 0 & 1 & 0 & 1 \\
\hline Hepatic (ALT >3x ULN) & 2 & 6 & 4 & 12 \\
\hline
\end{tabular}

* One case each in this group finally dropped out.

confirmed cases of typhoid fever. Twice as many were noted in both groups for all of the patients studied.

There was no deteriorization noted in the hematologic and renal function tests after treatment in both groups.

\section{Discussion}

Almost all fluoroquinolones after their introduction in Indonesia, were studied for their clinical and bacteriological efficacy in typhoid fever patients. It was not before long that levofloxacin the stereoisomer of ofloxacin with proven excellent antimicrobacterial activity in vitro againt $S$. typhi and excellent propertiesfor entering body fluids and organs and even cellular components harbouring the $S$. typhi bacteria could be recognized as probably an excellent alternative for treatment of typhoid fever $[15,16]$. Studies as early as this drug was introduced in the market already showed a high clinical success rate in treatment of typhoid fever as reported during the International Society of Chemotherapy Congress in Sydney Australia [17].

Almost all studies with its stereoisomer counterpart that is known as ofloxacin gave excellent results for treatment of typhoid fever in Indonesia as well as in other parts of South East Asia [18,19].

Our own open study confirmed the potential of levofloxacin to be used for typhoid fever by reaching very fast resolution of fever, the fasted ever recorded for a fluoroquinolone used for typhoid fever in the world. A pilot study showed that it only took on average two and a half day for a patient to become a febrile in hospital admitted uncomplicated typhoid fever cases treated by 500 mg levofloxacin once daily for 7 days (Nelwan et al., 2006) [10].

This comparative follow up study once again confirmed our initial study findings that this compound is not only equal but perhaps better than the existing treatment option for the typhoid fever patient. In this study the average resolution of fever in the levofloxacin group was significantly faster than in the ciprofloxacin treated patients.

It was interesting to note that none of the cases in both arms of treatment developed severe complication like intestinal bleeding or perforation. During the aftermath of treatment there were thrice as many increases in the ciprofloxacin group for liver transaminases where it increased more than three fold above the baseline values.

As was proclaimed that levofloxacin was one of the safest and the most tolerable fluoroquinolone [20,21], this study also could confirm the fact that there were less adverse reaction in the levofloxacin treated patient compared to the ciprofloxacin group. This seems to be a very significant finding and underscores the role levofloxacin may play in the typhoid fever patient where the gastrointestinal tract targetted by the microorganism is very sensitive.

Besides the adverse reactions in the cases with confirmed typhoid fever for which the figures were already given in the left side of Table 5 a total of 35 cases in the whole group receiving ciprofloxacin experienced adverse reactions and were the main cause of drop outs of the study (right side of Table 5). For all the patient treated with levofloxacin there were 20 cases with adverse reactions but none dropped out of the study, which was also a very significant finding favoring usage of levofloxacin. As final results of culture and sensitivity test took one week to complete, we are able to follow up all the patients until they finished their medication schedule.

The very early reports about fluoroquinolones used in Indonesia for uncomplicated typhoid fever denied relapses or carrier states [22-24] but in this study both arms definitely showed clinical relapse one in the levofloxacin group where $S$. typhi could not be confirmed bacteri- 
ology during the relapse and two cases in the ciprofloxacin group where $S$. typhi and $S$. arizonae could be identified. Whether this is a sign of increasing resistance against fluoroquinolones should be investigated further.

Finally it may be concluded that a 7 days oral regimen of levofloxacin $500 \mathrm{mg}$ daily for one week compared to two times $500 \mathrm{mg}$ ciprofloxacin daily for one week in typhoid fever in Indonesia showed faster fever clearance, and less adverse reactions clinically as well as laboratory for levofloxacin compared to ciprofloxacin.

\section{Acknowledgements}

The authors want to express their appreciation for providing funding and medication for the study to Daiichi Sankyo offices in Jakarta, Hong Kong, and Japan as well as Kalbe Farma Head Office in Jakarta Indonesia.

\section{REFERENCES}

[1] J. A. Crump, S. P. Luby and E. D. Mintz, "The Global Burden of Typhoid Fever," Bull World Health Organization, 2004, pp. 346-353.

[2] R. Chandra, S. Srinivasan, P. Nalini and R. S. Rao, "Multidrug Resistant Enteric Fever," Journal of Tropical Medicine \& Hygiene, Vol. 95, 1992, pp. 284-287.

[3] E. Braunwald, A. S. Fauci, D. L. Kasper, S. L. Hauser, D. L. Longo and J. L. Jameson, "Harrison's Manual of Medicine," McGraw-Hill, New York, 2012.

[4] E. J. Threlfall and L. R.Ward, "Decreased Susceptibility of Ciprofloxacin in Salmonella Enterica Serovar Typhi," Emerging Infectious Diseases, Vol. 7, 2001, pp. 448-450.

[5] K. Hirose, K. Tamura, H. Sagara and H. Watanabe, "Antibiotic Susceptibility of Salmonella Enterica Serovar Paratyphi A Isolated from Patients in Japan," Journal of Antimicrobial Chemotherapy, Vol. 45, No. 3, 2001, pp. 956-958. doi:10.1128/AAC.45.3.956-958.2001

[6] T. Dimitrov, E. E. Udo, O. Albaksami, S. Al-Shehab, A. Kilani, M. Shehab and A. Al-Nakkas, "Clinical and Microbiological Investigation of Typhoid Fever in an Infectious Disease Hospital in Kuwait," Journal of Antimicrobial Chemotherapy, Vol. 56, No. 4, 2007, pp. 538-544. doi:10.1099/jmm.0.46814-0

[7] R. H. H. Nelwan, "Ciprofloxacin Resistance Typhoid Fever in Indonesia," 10th Edition, Congress of Tropical Malaria, Nagasaki, 1996.

[8] S. Chitnis, V. Chitnis, N. Hemvani and D. S. Chitnis, "Ciprofloxacin Therapy for Typhoid Fever Needs Reconsideration," Journal of Infection and Chemotherapy, Vol. 12 , No. 6, 2006, pp. 402-404. doi:10.1007/s10156-006-0472-9

[9] H. A. Vinh, J. Wain, T. N. Vo, N. N. Cao, T. C. Mai and D. Bethell, "Two or Three Days of Ofloxacin Treatment for Uncomplicated MDR Typhoid Fever in Children," Antimicrobial Agents and Chemotherapy, Vol. 40, No. 4, 1996, pp. 958-961.

[10] R. H. H. Nelwan, K. C. Lie and N. D. Paramita, "Open
Study on Efficacy and Safety of Levofloxacin in the Treatment of Uncomplicated Typhoid Fever," Journal of Tropical Medicine, Vol. 37, 2006, pp. 126-130.

[11] R. H. H. Nelwan, "Clinical Score for Diagnosis of Typhoid Fever," Majalah Dokter Keluarga, Vol. 10, No. 4, 1991, pp. 17-21.

[12] A. Haque, N. Ahmed, A. Peerzada, A. Raza, S. Bashir and G. Abbas, "Utility of PCR in Diagnostic of Problematic Cases of Typhoid," Japanese Journal of Infectious Diseases, Vol. 54, 2001, pp. 237-239.

[13] M. Levine, O. Grados and R. H. Gilman, "Diagnostic Value of the Widal Test in Area Endemic for Typhoid Fever," American Journal of Tropical Medicine and Hygiene, Vol. 27, 1978, pp. 795-810.

[14] T. Pang and S. D. Puthucheary, "Significance of Widal Test in the Diagnosis of Typhoid Fever in an Endemic Area," Journal of Clinical Pathology, Vol. 36, No. 4, 1983, pp. 471-475. doi:10.1136/jcp.36.4.471

[15] R. Davis and H. M. Bryson, "Levofloxacin: A Review of Its Antibacterial Activity, Pharmacokinetics and Therapeutic Efficacy," Drugs, Vol. 47, No. 4, 1994, pp. 677710. doi:10.2165/00003495-199447040-00008

[16] K. F. Croom and K. L. Goa, "Levofloxacin: A Review of Its Use in the Treatment of Bacterial Infections," Pharmacokinetic Properties, Drugs, Vol. 64, 2003, pp. 27802782.

[17] J. Zheng, Z. Yuy and M. G. Wei, "Clinical Study in the Evaluation of Levofloxacin in the Treatment of Typhoid and Paratyphoid Fever," ISC Congress, Sydney, No. 3310, 1997, p. 100.

[18] R. H. H. Nelwan, H. I. Zulkarnain, J. Gunawan, Y. Supandiman and H. Yusuf, "A Comparative Study of Short Course Ciprofloxacin Treatment in Typhoid and Paratyphoid Fever," Drugs, Vol. 49, No. S2, 1995, pp. 463465. doi:10.2165/00003495-199500492-00136

[19] M. Velmonte, A. David and M. Bernardo, "Randomized Controlled Safety and Efficacy Study Comparing Ofloxacin with Chloramphenicol in the Treatment of Salmonella Enteric Fever," World Scientific Co., Singapore City, 1992.

[20] B. A. Lipsky and C. A. Baker, "Fluoroquinolones Toxicity Profile," Clinical Infectious Diseases, Vol. 28, No. 2, 1999, pp. 352-364. doi:10.1086/515104

[21] K. A. Rodvald, "Clinical Safety Profile of Fluoroquionolones," Penetration, 2006, pp. 34-40.

[22] R. H. H. Nelwan, A. W. Hendarwanto, I. Zulkarnain and J. Gunawan, "Double-Blind Randomized Controlled Study of Once Daily $400 \mathrm{mg}$ Pefloxacin in Typhoid and Paratyphoid Fever," Medical Journal of the Univiversity of Indonesia, Vol. 3, 1994, pp. 38-41.

[23] R. H. H. Nelwan, B. W. Hendarwanto, I. Zulkarnain and J. Gunawan, "A Controlled Study of Ofloxacin in Typhoid Fever," Majalah Kesehatan Masyarakat Indonesia, Vol. 22, 1995, pp. 58- 65.

[24] R. H. H. Nelwan, B. Setiawan, J. Gunawan and H. I. Zulkarnain, "Short Course Fleroxacin in Typhoid Fever," Acta Medica Indonesia, Vol. 30, 1998, pp. 25-30. 